\title{
ADAPTABILIDAD DE POBLACIONES DE Pinus pinceana EN DOS SUELOS
}

\author{
ADAPTABILITY OF Pinus pinceana POPULATIONS IN TWO SOILS
}

\author{
Diana Córdoba Rodríguez ${ }^{1,2}$, Jesús Vargas Hernández ${ }^{1 *}$ y José A. Arévalo Ramírez ${ }^{2}$
}

\begin{abstract}
${ }^{1 *}$ Programa Forestal, Campus Montecillo, Colegio de Postgraduados. Km 36.5 Carretera México-Texcoco. 56230, Montecillo, Texcoco, México. Tel. 5804-5900 ext. 1469, Fax: 5804-5956. ${ }^{2}$ Depto. El Hombre y su Ambiente, CBS, Universidad Autónoma Metropolitana-Unidad Xochimilco. Calzada del Hueso 1100. 04960, México, D. F.

\section{RESUMEN}

Pinus pinceana se encuentra sometida a fuertes presiones ambientales y antrópicas que la declinan hacia su extinción, y existe poca información ecológica y genética sobre esta especie, en particular sobre las posibles diferencias entre poblaciones en el crecimiento y la distribución de biomasa en plántulas, como mecanismos adaptativos a condiciones ambientales. En este estudio se determinó el patrón de distribución de biomasa y la tasa de crecimiento de la raíz como características adaptativas en 10 poblaciones naturales de este pino provenientes de las regiones norte y sur de la Sierra Madre Oriental de México, establecidas en rizotrones que contenían dos tipos de suelo. Las condiciones del suelo afectaron de manera significativa $(P<0.05)$ el crecimiento y la asignación de recursos a la raíz. Las plantas de la región sur acumularon más biomasa total que las del norte, pero no hubo diferencias $(P=\mathbf{0 . 0 5})$ entre poblaciones en el patrón de asignación de biomasa. La especie mostró amplia plasticidad fenotípica en el crecimiento de raíz en respuesta a las condiciones edáficas, lo cual puede ser un mecanismo evolutivo para aprovechar las esporádicas condiciones ambientales favorables en su hábitat natural.

Palabras clave: Pinus pinceana, condiciones edáficas, distribución de biomasa, plasticidad fenotípica.

\section{SUMMARY}

Strong environmental and human-derived pressures are driving Pinus pinceana towards extinction, and very little ecologic and genetic information is available about this species, particularly on population variation in seedling growth and biomass distribution as adaptive mechanisms to environmental conditions. In this study, biomass distribution and root growth rate were determined for 10 natural populations of $\boldsymbol{P}$. pinceana brought from the northern and southern region of Sierra Madre Oriental in México, and grown in rizotrons with soil from two regions. Seedling growth and biomass allocation to roots were significantly $(P<0.05)$ affected by soil conditions. Seedlings from the southern region accumulated more biomass than those from the northern region, but no differences $(P$ $=0.05$ ) were found among populations in biomass allocation to roots. A broad phenotypic plasticity of this species in root growth was found in the two soils, possibly as an evolutionary mechanism to take advantage of the sporadic favorable environmental conditions in its natural habitat.
Index words: Pinus pinceana, soil conditions, biomass distribution, phenotypic plasticity.

\section{INTRODUCCIÓN}

Pinus pinceana Gordon es un pino piñonero que crece en poblaciones dispersas y aisladas, localizadas en dos grandes regiones a lo largo de la Sierra Madre Oriental en los Estados de Hidalgo, Querétaro, San Luis Potosí, Zacatecas y Coahuila (Perry, 1991; Ledig et al., 2001). Estas regiones están separadas por montañas y grandes extensiones de zonas áridas; además, dentro de cada región, las poblaciones de Pinus pinceana están aisladas unas de otras por barreras geográficas, lo que dificulta el intercambio genético entre ellas. El aislamiento genético favorece la diferenciación de las poblaciones por efectos de deriva genética, como se ha demostrado en estudios de diversidad genética de la especie mediante isoenzimas (Ledig et al., 2001; Molina-Freaner et al., 2001). Las diferencias ambientales entre regiones sugieren la posibilidad de que la selección natural haya ocasionado diferencias entre las poblaciones en características de importancia adaptativa a factores específicos de estrés ambiental.

Existe poca información ecológica y génetica de la especie, a pesar de que se encuentra bajo fuertes presiones ambientales y antrópicas que la orientan hacia su extinción (Molina-Freaner et al., 2001). En particular se carece de datos sobre crecimiento y distribución de biomasa en etapas tempranas del desarrollo de las plántulas, como posibles mecanismos adaptativos a condiciones ambientales locales. Dado que las propiedades físicas y químicas del suelo afectan la tasa de crecimiento y desarrollo de las raíces (Pritchett, 1991) y la asignación de biomasa entre órganos de la planta, es posible que exista variación entre las poblaciones de la 
especie en respuesta a las condiciones del suelo. Por ejemplo, Keyes y Grier (1981) encontraron diferencias en la asignación de biomasa a la raíz y al tallo en árboles de Pseudotsuga menziesii al crecer en sitios con diferentes niveles de productividad. Estudios con diferentes especies leñosas muestran que el patrón de asignación de recursos en las plantas depende de las condiciones del suelo, como fertilidad y disponibilidad de agua, así como de las características genéticas intrínsecas (Koslowski et al., 1991; Li et al., 1991; Zhang et al., 1996; Wang et al., 1998; Barnes, 2002; Chan et al., 2003). Las diferencias en crecimiento y asignación de biomasa a la raíz explican en gran medida las diferencias en crecimiento y productividad entre poblaciones, así como en la capacidad de adaptación a condiciones de estrés (Abrams et al., 1990; Zhang et al., 1996).

Las pruebas de progenie evalúan el desempeño de plántulas que crecen en condiciones ambientales similares, y por ello constituyen una herramienta importante en el análisis del componente ambiental y genético de las características cuantitativas con valor adaptativo (ElKassaby, 1991). Este tipo de evaluaciones ha demostrado que generalmente existen niveles altos de variación intere intrapoblacional del género Pinus (Kormanik et al., 1990; Li et al., 1991), aunque también existen excepciones importantes. En Pinus pinceana se desconoce la magnitud y la estructura de la diversidad genética en estos aspectos, que podrían ser de importancia crucial en el establecimiento inicial de las plántulas en condiciones naturales debido a la limitada disponibilidad de humedad en los ecosistemas donde habita esta especie.

Por lo anterior, el objetivo de este trabajo fue determinar las diferencias en crecimiento de raíces y distribución de biomasa en plántulas de 10 poblaciones naturales de Pinus pinceana, en dos condiciones edáficas, e identificar a las poblaciones con mayor capacidad de desarrollo inicial de la raíz y el grado de diferenciación geográfica entre ellas.

\section{MATERIALES Y MÉTODOS}

\section{Establecimiento del ensayo}

Se evaluaron 10 poblaciones naturales de Pinus pinceana que representan el área de distribución natural conocida de la especie; seis provienen de la región norte, en los Estados de Coahuila y Zacatecas, y cuatro de la región sur, en los Estados de San Luis Potosí, Querétaro e Hidalgo (Cuadro 1). De cada población se tomó una muestra de 50 semillas de polinización libre de dos árboles, bajo el supuesto de que en esas semillas están representados los alelos paternos de cada población. En dos poblaciones (El Recreo, Coah., y El Arenalito, Hgo.) también se colectaron muestras de suelo de los primeros $20 \mathrm{~cm}$ de profundidad para utilizarlos como sustratos.

Cuadro 1. Localización geográfica de las poblaciones de Pinus pinceana incluidas en el estudio.

\begin{tabular}{|c|c|c|c|c|}
\hline \multirow{2}{*}{ Núm. de control } & \multirow{2}{*}{ Población } & \multicolumn{2}{|c|}{ Coordenadas geográficas } & \multirow{2}{*}{$\begin{array}{l}\text { Altitud } \\
\text { (m) }\end{array}$} \\
\hline & & Latitud (N) & Longitud (O) & \\
\hline \multicolumn{5}{|l|}{ Región Norte } \\
\hline 1 & Norias, Coah. & $26^{\circ} 27^{\prime} 08^{\prime \prime}$ & $101^{\circ} 35^{\prime} 40^{\prime \prime}$ & 1800 \\
\hline 2 & El Recreo, Coah. & $25^{\circ} 17^{\prime} 40^{\prime \prime}$ & $101^{\circ} 00^{\prime} 02^{\prime \prime}$ & 2238 \\
\hline 3 & El Cinco, Coah. & $25^{\circ} 10^{\prime} 41^{\prime \prime}$ & $101^{\circ} 41^{\prime} 16^{\prime \prime}$ & 2250 \\
\hline 4 & Santa Elena, Coah. & $25^{\circ} 01^{\prime} 38^{\prime \prime}$ & $101^{\circ} 24^{\prime} 29^{\prime \prime}$ & 2077 \\
\hline 5 & Lomas El Orégano, Zac. & $24^{\circ} 30^{\prime} 19^{\prime \prime}$ & $101^{\circ} 27^{\prime} 48^{\prime \prime}$ & 2305 \\
\hline 6 & San José Carbonerillas, Zac. & $24^{\circ} 28^{\prime} 16^{\prime \prime}$ & $100^{\circ} 26^{\prime} 53^{\prime \prime}$ & 2300 \\
\hline \multicolumn{5}{|l|}{ Región Sur } \\
\hline 7 & Ejido Núñez, S. L. P. & $22^{\circ} 40^{\prime} 06^{\prime \prime}$ & $100^{\circ} 28^{\prime} 24^{\prime \prime}$ & 1930 \\
\hline 8 & Maguey Verde, Qro. & $21^{\circ} 05^{\prime} 25^{\prime \prime}$ & $099^{\circ} 42^{\prime} 00^{\prime \prime}$ & 2176 \\
\hline 9 & El Arenalito, Hgo. & $20^{\circ} 39^{\prime} 27^{\prime \prime}$ & $099^{\circ} 02^{\prime} 58^{\prime \prime}$ & 1880 \\
\hline 10 & San Cristóbal, Hgo. & $20^{\circ} 37^{\prime} 53^{\prime \prime}$ & $098^{\circ} 58^{\prime} 31^{\prime \prime}$ & 1915 \\
\hline
\end{tabular}


Las semillas se embebieron en una solución de peróxido de hidrógeno $\left(\mathrm{H}_{2} \mathrm{O}_{2}\right)$ a $1 \%$ durante $24 \mathrm{~h}$ y posteriormente se germinaron durante $72 \mathrm{~h}$ en una cámara de ambiente controlado a $24{ }^{\circ} \mathrm{C}$ (Lab-line Instrument Inc., Biotronette). Conforme emergió la radícula, las semillas se trasplantaron a rizotrones (cajas de madera de $60 \mathrm{~cm}$ de largo x $10 \mathrm{~cm}$ de ancho x $50 \mathrm{~cm}$ de alto, con una pared de cristal protegida por otra pared de madera movible) que contenían alguno de los dos tipos de suelo (10 con el de El Recreo, Coah. y 10 con el de El Arenalito, Hgo.). El suelo de $\mathrm{El}$ Arenalito tiene una textura de migajón arcillo-limoso que le confiere menor capacidad de retención de humedad que el suelo de El Recreo cuya textura es arcillosa. Además, el suelo de El Arenalito tiene menor contenido de materia orgánica y menor fertilidad, menor capacidad de intercambio catiónico (CIC) y valores más bajos de nitrógeno, fósforo, potasio y otros cationes intercambiables, en comparación con el suelo de El Recreo (Cuadro 2).

Cuadro 2. Principales características físicas y químicas de los suelos utilizados en el ensayo (El Recreo, Coah. y El Arenalito, Hgo.).

\begin{tabular}{lcc}
\hline Característica & El Recreo, Coah. & El Arenalito, Hgo. \\
\hline Textura & Arcillosa & Migajón arcillo-limosa \\
Contenido de humedad a & 41.90 & 27.70 \\
capacidad de campo (\%) & & \\
Contenido de materia & 15.30 & 6.84 \\
orgánica (\%) & & \\
pH & 7.71 & 7.88 \\
$\mathrm{CIC}\left(\right.$ meq $\left.100 \mathrm{~g}^{-1}\right)$ & 42.20 & 35.20 \\
$\mathrm{~N}$ total $(\%)$ & 0.72 & 0.30 \\
$\mathrm{P}\left(\mathrm{mg} \mathrm{kg}^{-1}\right)$ & 30.90 & 18.80 \\
$\mathrm{~K}\left(\mathrm{cmol} \mathrm{kg}^{-1}\right)$ & 0.43 & 0.34 \\
$\mathrm{Ca}^{++}\left(\mathrm{meq} \mathrm{L}^{-1}\right)$ & 17.50 & 10.00 \\
$\mathrm{Mg}^{++}\left(\mathrm{meq} \mathrm{L}^{-1}\right)$ & 3.60 & 1.40 \\
$\mathrm{~K}^{+}\left(\right.$meq L$\left.^{-1}\right)$ & 0.59 & 0.25 \\
$\mathrm{Na}^{+}\left(\mathrm{meq}^{-1}\right)$ & 1.70 & 0.46 \\
$\mathrm{HCO}_{3}\left(\mathrm{meq} \mathrm{L}^{-1}\right)$ & 13.30 & 6.67 \\
$\mathrm{SO}_{4}\left(\right.$ meq L L $\left.^{-1}\right)$ & 2.89 & 0.67 \\
$\mathrm{Conductividad} \mathrm{eléctrica}_{\left.(\mathrm{dS} \mathrm{m})^{-1}\right)}$ & 1.79 & 0.96 \\
\end{tabular}

Los tratamientos se distribuyeron en un diseño experimental en parcelas divididas y bloques completos al azar; en las parcelas grandes (constituidas por los rizotrones) se asignaron los tipos de suelo, con 10 repeticiones, y en cada una se trasplantó una semilla de cada población de Pinus pinceana (parcela chica), más dos semillas extras (provenientes de las mismas 10 poblaciones) que se colocaron en los extremos del rizotrón pera eliminar el efecto de orilla. Los rizotrones se mantuvieron en condiciones de vivero durante tres meses $(91 \mathrm{~d})$, con una humedad cercana a capacidad de campo, mediante riego cada $24 \mathrm{~h}$.

\section{Variables evaluadas}

A los tres meses de establecido el ensayo, las plantas se extrajeron para determinar la longitud de la parte aérea y de la raíz principal, así como el peso seco de acículas, tallo y raíz. Para ello, se separó la pared de cristal de los rizotrones y se eliminó el suelo con agua para extraer las raíces completas; se midió la longitud $(\mathrm{cm})$ de la parte aérea y de la raíz principal (más larga). Las partes de la planta se colocaron en bolsas de papel, se secaron en un horno eléctrico a $70{ }^{\circ} \mathrm{C}$ durante $48 \mathrm{~h}$, y se pesaron en una balanza de precisión (Ohaus Navigator), con aproximación de $0.01 \mathrm{~g}$. Con los datos de biomasa de cada planta se determinó la relación raíz/parte aérea (RPA), en porcentaje:

$$
\operatorname{RPA}(\%)=\frac{[\mathrm{psr}][100]}{\text { [pspa] }}
$$

Donde: psr $=$ peso seco de la raíz, en gramos; y pspa $=$ peso seco de la parte aérea de la planta, en gramos.

\section{Análisis estadístico}

Para determinar el efecto del tipo de suelo, así como las posibles diferencias en crecimiento asociadas con las regiones y las poblaciones dentro de regiones, se llevó a cabo un análisis de varianza conforme al arreglo en parcelas divididas, mediante el paquete estadístico SAS (SAS Institute, 1987) de acuerdo con el siguiente modelo, que considera a las poblaciones anidadas dentro de regiones:

$\mathrm{Y}_{\mathrm{ij} j \mathrm{k}}=\mu+\mathrm{B}_{\mathrm{i}}+\mathrm{S}_{\mathrm{j}}+\mathrm{BS}_{\mathrm{ij}}+\mathrm{R}_{\mathrm{k}}+\mathrm{SR}_{\mathrm{jk}}+\mathrm{P}_{\mathrm{l}(\mathrm{k})}+\mathrm{SP}_{\mathrm{j}(\mathrm{k})}+\varepsilon_{\mathrm{ij} \mathrm{k}}$

Donde: $Y_{\mathrm{ijjk}}$ es el valor de la características medida en la planta de la i-ésima repetición en el j-ésimo suelo y la lesíma población de la k-ésima región geográfica; $\mu$ es la media del experimento para la característica evaluada; $\mathrm{B}_{\mathrm{i}}$ es el efecto del i-ésimo bloque (repetición); $S_{\mathrm{j}}$ es el efecto del j-ésimo suelo; $\mathrm{BS}_{\mathrm{ij}}$ es el efecto de interacción entre el i-ésimo bloque y el j-ésimo suelo (error de parcela grande); $\mathrm{R}_{\mathrm{k}}$ es el efecto de la k-ésima región geográfica; $\mathrm{SR}_{\mathrm{jk}}$ es el efecto de la interacción entre el j-ésimo suelo y la k-ésima región geográfica; $\mathrm{P}_{1(\mathrm{k})}$ es el efecto de la 1 ésima población dentro de la k-ésima región geográfica; $\mathrm{SP}_{\mathrm{j}(\mathrm{k})}$ es el efecto de interacción entre el j-ésimo suelo y la 1-ésima población dentro de la k-ésima región geográfica; y $\varepsilon_{\mathrm{j} j \mathrm{jk}}$ es el error experimental.

En los casos en que hubo diferencias significativas entre poblaciones, se hizo una comparación de medias con la prueba de comparaciones múltiples de Duncan $(\alpha=$ 
0.05). Para estimar el grado de plasticidad de las poblaciones con referencia al crecimiento de las raíces y a la asignación de biomasa en respuesta a los dos tipos de suelo, se calculó la correlación entre los valores promedio por población de estas características en los dos ambientes de crecimiento, así como el cambio relativo en las características promedio de las plantas al pasar de un ambiente a otro.

\section{RESULTADOS Y DISCUSIÓN}

\section{Efecto del suelo}

El tipo de suelo tuvo fuerte influencia en el crecimiento de Pinus pinceana, especialmente de la raíz. Con excepción de longitud y peso seco de la parte aérea, el suelo afectó de manera significativa $(\mathrm{P}<0.05)$ al crecimiento y distribución de materia seca en las plántulas (Cuadro 3). En las variables donde hubo diferencias significativas, las plantas que crecieron en el suelo de El Recreo, Coah. mostraron los valores más bajos (Cuadro 4). La raíz, por ejemplo, en el suelo de El Arenalito, Hgo., creció 50 \% más en longitud y acumulación de biomasa que en el de El Recreo, Coah. Este resultado muestra que las diferencias en las características edáficas repercutieron en el crecimiento radical, a pesar del corto tiempo de evaluación. Este impacto del suelo es importante, porque en la raíz se reflejan de manera inmediata las características que le confieren ventajas adaptativas a los individuos, para la obtención de recursos y tolerar los efectos de factores físicos y bióticos adversos (Yoav et al., 1993).

Dado que el suelo de El Recreo, Coah. tenía mejor fertilidad (Cuadro 2), es posible que el menor crecimiento de raíz observado en este suelo se deba principalmente a sus características físicas. Un suelo arcilloso generalmente contiene un menor contenido de oxígeno y presenta mayor grado de compactación, lo que dificulta el crecimiento de la raíz. Según Koslowski et al. (1991) y Yoav et al. (1993), la presión ejercida por un suelo arcilloso sobre la raíz es suficiente para impedir su desarrollo adecuado; en cambio, una textura de migajón arcillo-limoso como la del suelo de El Arenalito, Hgo., con mayor porosidad, permite mayor penetración y crecimiento al sistema radical.

Cuadro 3. Cuadrados medios y significancia estadística para características de crecimiento de plantas de diez poblaciones naturales de Pinus pinceana en dos tipos de suelo.

\begin{tabular}{|c|c|c|c|c|c|c|}
\hline \multirow{2}{*}{ Variable } & \multicolumn{6}{|c|}{ Cuadrados medios } \\
\hline & Suelo (S) & Región (R) & $\mathrm{S} \times \mathrm{R}$ & Población (P) & $S \times P$ & Error \\
\hline Longitud de planta & 6.85 & $11.42 * *$ & 0.96 & $3.66^{* *}$ & 1.13 & 0.96 \\
\hline Longitud de raíz & $5566.84 * *$ & 17.06 & 215.94 & 205.31 & $231.50 *$ & 113.54 \\
\hline Peso seco de planta & $0.11 *$ & $0.03 *$ & $<0.01$ & $0.01 *$ & 0.01 & $<0.01$ \\
\hline Peso seco de parte aérea & 0.04 & $0.02 *$ & $<0.01$ & $<0.01 *$ & $<0.01$ & $<0.01$ \\
\hline Peso seco de raíz & $0.02 * *$ & $<0.01$ & $<0.01$ & $<0.01$ & $<0.01$ & $<0.01$ \\
\hline Relación raíz/parte aérea & $0.22 *$ & 0.03 & $<0.01$ & $<0.01$ & $<0.01$ & 0.01 \\
\hline
\end{tabular}

* $\mathrm{y} * *$ Diferencias significativas con $\mathrm{P} \leq 0.05$ y $\mathrm{P} \leq 0.01$. Grados de libertad: $\mathrm{S}=1 ; \mathrm{R}=1 ; \mathrm{S} \times \mathrm{R}=1 ; \mathrm{P}=9 ; \mathrm{S} \times \mathrm{P}=8$.

Cuadro 4. Características de crecimiento de plantas de Pinus pinceana en dos tipos de suelo y de dos regiones geográficas, en promedio de 10 poblaciones.

\begin{tabular}{|c|c|c|c|c|}
\hline Característica & \multicolumn{2}{|c|}{ Tipo de suelo } & \multicolumn{2}{|c|}{ Regiones geográficas } \\
\hline Longitud de planta $(\mathrm{cm})$ & $5.66 \mathrm{a}^{\dagger}$ & $5.24 \mathrm{a}$ & $5.18 \mathrm{~b}^{\dagger}$ & $5.71 \mathrm{a}$ \\
\hline Peso seco de planta (g) & $0.21 \mathrm{~b}$ & $0.25 \mathrm{a}$ & $0.21 \mathrm{~b}$ & $0.24 \mathrm{a}$ \\
\hline Peso seco de parte aérea (g) & $0.15 \mathrm{a}$ & $0.18 \mathrm{a}$ & $0.16 \mathrm{~b}$ & $0.18 \mathrm{a}$ \\
\hline
\end{tabular}

${ }^{\dagger}$ Valores promedio entre tipos de suelo o regiones geográficas seguidos por la misma letra no son estadísticamente diferentes $(\mathrm{P} \leq 0.05)$. 
Las diferencias entre los dos suelos en el crecimiento de la raíz y en el patrón de asignación de biomasa entre los órganos, también pueden explicarse por diferencias en la fertilidad de los suelos. En otras especies leñosas se ha encontrado que un mecanismo de plasticidad fenotípica de las plantas ante suelos poco fértiles, es asignar más biomasa a la raíz que en plantas que crecen en suelos fértiles o en condiciones ambientales más favorables y no sujetas a estrés (Rajkai, 1991; Li et al., 1991; Wang et al., 1998; Chan et al., 2003). Se ha estimado que algunas coníferas invierten más de $60 \%$ de su productividad primaria neta en la formación de raíces para asegurar el abastecimiento de agua y nutrimentos, y la supervivencia en sitios pobres (Kozlowski et al., 1991; Cinnirella et al., 2002). García y Vargas (2000) encontraron, por ejemplo, mayor cantidad de materia seca radical en varias poblaciones de Gliricidia sepium que crecían en condiciones de estrés hídrico, que sin estrés.

\section{Efecto de la procedencia geográfica}

A diferencia de lo que ocurrió con el tipo de suelo, la región geográfica de origen del germoplasma no tuvo efecto significativo en el crecimiento de raíz, aunque sí sobre el crecimiento de la parte aérea y la acumulación de peso seco en toda la plántula (Cuadro 3). La interacción suelo x región no fue significativa para variable alguna, lo cual indica que el efecto del tipo de suelo fue similar sobre el comportamiento promedio de las poblaciones en ambas regiones. Sin embargo, las plantas de la región sur alcanzaron una altura $10 \%$ mayor y acumularon $13 \%$ más biomasa aérea y total que las plantas de la región norte, pero tuvieron una longitud y biomasa de raíz similar entre ellas (Cuadro 4). Tales diferencias en el crecimiento de la parte aérea entre las dos regiones no fueron suficientes para que se reflejaran en diferencias significativas en el patrón de distribución de biomasa entre órganos.

Las poblaciones de Pinus pinceana provenientes de la región sur tuvieron un mayor potencial de crecimiento, aún cuando crecieron en el suelo de la región norte y en un corto periodo de evaluación (tres meses), lo cual sugiere que poseen una carga genética diferente, probablemente asociada con mecanismos de plasticidad para responder a condiciones ambientales menos restrictivas, que le permite aprovechar condiciones favorables esporádicas en su hábitat natural. Salazar et al. (1999) también encontraron amplia variación en la velocidad de crecimiento entre poblaciones de varias especies mexicanas de Pinus ( $P$. greggii, $P$. patula, $P$. tecunumanii y $P$. maximinoi) al crecer en un mismo sitio, lo cual indica que las diferencias se deben principalmente a la carga genética de cada población.
Parece ser que las diferencias regionales en productividad potencial se obtienen sin modificar el patrón de asignación de biomasa a la raíz, por lo que debe haber otros mecanismos fisiológicos que expliquen la mayor tasa de crecimiento inicial de las plantas de la región sur; también es posible que con un mayor tiempo de evaluación se manifiesten de manera clara las diferencias en la distribución de biomasa a las raíces entre las plantas de las dos regiones geográficas.

\section{Diferencias entre poblaciones}

Al igual que en el caso de las regiones geográficas, sólo se encontraron diferencias significativas $(\mathrm{P}<0.05)$ entre poblaciones (dentro de las regiones geográficas) en el crecimiento y acumulación de biomasa de la parte aérea y total de la plántula, pero no en el crecimiento de la raíz (Cuadro 3). Sin embargo, en la longitud de la raíz se encontró un efecto significativo $(\mathrm{P}<0.05)$ de la interacción entre poblaciones por tipo de suelo, lo cual indica que las poblaciones de una región geográfica varían en esta característica al crecer en cada uno de los suelos.

La variación entre poblaciones de una misma región geográfica en el crecimiento de la parte aérea (Cuadro 5) muestra que en la región norte destaca la población 4 proveniente de Santa Elena, Coah. por tener los valores más bajos de biomasa aérea y total, mientras que la Población 6 de San José Carbonerillas, Zac. da los valores más altos, con más de $40 \%$ en biomasa total, pero mantiene una relación raíz/parte aérea similar a las demás. En la región sur, las Poblaciones 7 (Ejido Nuñez, S. L. P.) y 9 (El Arenalito, Hgo.) presentaron los valores extremos en biomasa aérea y total, con diferencias similares a las encontradas entre las poblaciones de la región norte.

La falta de significancia estadística en la relación raíz/parte aérea entre poblaciones puede indicar que Pinus pinceana tiene una baja flexibilidad genética en el patrón de asignación de biomasa, quizás como resultado de procesos de adaptación a condiciones ambientales de sequía extrema en donde ha evolucionado, como se ha señalado para otras especies leñosas (Alscher y Cumming, 1990; Bridgwater, 1990), a pesar de la variación genética de la especie en la productividad de etapas iniciales de establecimiento en campo. También es posible que se requiera un mayor periodo de evaluación para que se manifiesten de manera clara las diferencias en la asignación de biomasa entre las poblaciones. De hecho, la interacción poblaciones $\mathrm{x}$ tipo de suelo para crecimiento en longitud de la raíz principal muestra que la respuesta de las plantas no es simple, ya que no hay una respuesta homogénea en el crecimiento radical al cambiar el suelo. 
Cuadro 5. Características de crecimiento en plantas de Pinus pinceana originarias de 10 poblaciones naturales, en promedio de dos tipos de suelo.

\begin{tabular}{|c|c|c|c|c|c|c|c|c|c|c|}
\hline \multirow[b]{2}{*}{ Característica $^{\dagger}$} & \multicolumn{6}{|c|}{ Poblaciones de la región norte } & \multicolumn{4}{|c|}{ Poblaciones de la región sur } \\
\hline & 1 & 2 & 3 & 4 & 5 & 6 & 7 & 8 & 9 & 10 \\
\hline $\operatorname{lr}(\mathrm{cm})$ & 27.70 & 24.42 & 27.02 & 22.72 & 25.63 & 33.53 & 22.73 & 26.42 & 26.04 & 29.57 \\
\hline pspa (g) & $0.17 \mathrm{abc}$ & $0.16 \mathrm{bc}$ & $0.17 \mathrm{abc}$ & $0.13 \mathrm{c}$ & $0.16 \mathrm{bc}$ & $0.18 \mathrm{ab}$ & $0.14 \mathrm{bc}$ & $0.19 a b$ & $0.20 \mathrm{a}$ & $0.20 \mathrm{a}$ \\
\hline psr (g) & 0.06 & 0.06 & 0.06 & 0.04 & 0.05 & 0.07 & 0.04 & 0.06 & 0.06 & 0.07 \\
\hline
\end{tabular}

$\$$ Promedios en una misma característica seguidos por la misma letra no son estadísticamente diferentes (Duncan, 0.05).

${ }^{\dagger} \mathrm{lp}=$ longitud de planta; $\mathrm{lr}=$ longitud de raíz; psp = peso seco de planta; pspa = peso seco de parte aérea; psr = peso seco de raíz; rpa = relación raíz/parte aérea. Código de las poblaciones: 1) Norias, Coah.; 2) El Recreo, Coah.; 3) El Cinco, Coah.; 4) Santa Elena, Coah.; 5) Lomas El Orégano, Zac.; 6) San José Carbonerillas, Zac.; 7) Ejido Nuñez, S.L.P.; 8) Maguey Verde, Qro.; 9) El Arenalito, Hgo.; 10) San Cristóbal, Hgo.

Por ejemplo, debido a las diferencias en las características del suelo, se esperaba que todas las poblaciones de la región norte aumentaran en cerca de 50 $\%$ la longitud de raíz al crecer en el suelo del Estado de Hidalgo (Cuadro 4), pero los aumentos variaron desde apenas $10 \%$ en la población de Lomas El Orégano, Zac., hasta más de $100 \%$ en las poblaciones de El Cinco, Coah. y San José Carbonerillas, Zac. (Cuadro 6). Por las mismas razones, se esperaba que las poblaciones del sur redujeran en alrededor de $35 \%$ la longitud de la raíz al crecer en el suelo de Coahuila (Cuadro 4), pero la reducción varió desde $21 \%$ en la población de San Cristóbal, Hgo. hasta casi $50 \%$ en la de Maguey Verde, Qro. (Cuadro 6). Esto demuestra que las poblaciones difieren en el grado de sensibilidad o plasticidad de la raíz al modificarse las condiciones del suelo con respecto al de su región de origen. Así, mientras la población de Lomas El Orégano, Zac. no tiene la capacidad de aprovechar las posibilidades de una mayor longitud de raíz al crecer en el suelo de Hidalgo, las Poblaciones de El Cinco, Coah. y de San José Carbonerillas, Zac. aprovechan al máximo tales condiciones favorables de suelo.

La variación entre poblaciones en algunas características del crecimiento y desarrollo en respuesta a los dos suelos (Cuadros 5 y 6) muestra que dentro de Pinus pinceana, cuya área de distribución es relativamente restringida y las poblaciones están aisladas entre sí, existen diferencias genéticas importantes, lo que confirma la necesidad de conservar los recursos genéticos de sus poblaciones naturales presentes en las regiones geográficas que habita. Aunque no se encontraron diferencias significativas entre las poblaciones en el patrón de asignación de recursos, es evidente que hay diferencias importantes entre ellas en la capacidad adaptativa al tipo de suelo, desde el punto de vista del crecimiento de la raíz. Por ejemplo, en las dos regiones geográficas se detectó variación importante en el crecimiento longitudinal de la raíz al crecer en el otro suelo.
No se encontró correlación significativa $(\mathrm{P} \leq 0.05)$ entre los dos suelos en los valores promedio de las poblaciones para longitud y peso de raíz, ni en la relación parte aérea-raíz, lo cual indica que para estas características $P$. pinceana es una especie plástica desde el punto de vista fenotípico. Tal plasticidad fenotípica puede representar una ventaja para ajustar su patrón de crecimiento a las condiciones naturales en su hábitat y para enfrentar condiciones adversas inesperadas que de otra manera podrían ser perjudiciales para su supervivencia; al mismo tiempo, la plasticidad le permite aprovechar condiciones ambientales favorables para lograr una mayor productividad.

Cuadro 6. Longitud promedio de raíz en plantas de 10 poblaciones de Pinus pinceana, al crecer en dos tipos de suelo.

\begin{tabular}{lccc} 
& \multicolumn{3}{c}{ Longitud de raíz $(\mathrm{cm})$} \\
\cline { 2 - 4 } & $\begin{array}{c}\text { El Recreo, } \\
\text { Coah. } \\
\left(\mathrm{S}_{1}\right)\end{array}$ & $\begin{array}{c}\text { El Arenalito, } \\
\text { Hgo. } \\
\left(\mathrm{S}_{2}\right)\end{array}$ & $\mathrm{S}_{2} / \mathrm{S}_{1}$ \\
\hline Región norte & & & \\
Norias, Coah. & 18.93 & 36.47 & 1.93 \\
El Recreo, Coah. & 19.35 & 29.49 & 1.52 \\
El Cinco, Coah. & 16.43 & 37.62 & 2.29 \\
Santa Elena, Coah. & 18.05 & 27.40 & 1.52 \\
Lomas El Orégano, Zac. & 24.34 & 26.92 & 1.11 \\
San José Carbonerillas, Zac. & 21.82 & 45.25 & 2.07 \\
& & & \\
Región sur & & & \\
Ejido Núñez, S. L. P. & 19.61 & 25.86 & 1.32 \\
Maguey Verde, Qro. & 18.44 & 34.40 & 1.87 \\
El Arenalito, Hgo. & 21.74 & 30.34 & 1.40 \\
San Cristóbal, Hgo. & 26.11 & 33.02 & 1.26 \\
\hline
\end{tabular}

\section{CONCLUSIONES}

El tipo de suelo afecta el crecimiento de la raíz de plántulas de Pinus pinceana, pues modifica la tasa de crecimiento y el patrón de asignación de biomasa. Existe una diferenciación morfológica entre las plantas de las dos regiones geográficas de origen (norte y sur) y entre las 
poblaciones dentro de cada región, especialmente en el crecimiento de la parte aérea. Si bien la especie tiene un patrón de crecimiento definido, cuenta con plasticidad fenotípica, especialmente en el crecimiento de raíz, en respuesta a las condiciones ambientales representadas por los dos suelos. Esta plasticidad puede ser un mecanismo de ajuste a condiciones adversas inesperadas o a la presencia esporádica de condiciones ambientales favorables para su crecimiento. Por tanto, un programa de conservación de la especie debe incluir poblaciones de las dos regiones geográficas en su área de distribución natural.

\section{BIBLIOGRAFÍA}

Abrams M D, M E Kubiske, K C Steiner (1990) Drought adaptations and response in five genotypes of Fraxinus pennsylvanica Marsh.: photosynthesis, water relations and leaf morphology. Tree Physiol. 6:305-315.

Alscher G R, R J Cumming (1990) Stress responses in plants: adaptation and acclimation mechanisms. Wiley-Liss. New York. 407 p.

Barnes A D (2002) Effects of phenology, water availability and seed source on loblolly pine biomass partitioning and transpiration. Tree Physiol. 22: 733-740.

Bridgwater F E (1990) Shoot elongation patterns of loblolly pine families selected for contrasting growth potencial. For. Sci. 36: 641-656.

Chan S S, S R Radosevich, A T Grotta (2003) Effects of contrasting light and soil moisture availability on the growth and biomass allocation of Douglas-fir and red alder. Can. J. For. Res. 33:106-117.

Cinnirella S, F Magnani, A Saracino, M Borghetti (2002) Response of a mature Pinus laricio plantation to a three-year restriction of water supply: structural and functional acclimation to drought. Tree Physiol. 22:21-30.

El-Kassaby A Y (1991) Genetic variation within and among conifer populations: Review and evaluation of methods. In: M E Fineschi, H Malvolti, F Cannata, H H Hattemer (eds). Biochemical Markers in the Population Genetics of Forest Trees. SPD Acad. Publ. by the Hague, The Netherlands. pp:61-76.

García F M, J J Vargas H (2000) Growth and biomass allocation of Gliricidia sepium seed sources under drought conditions. J. Sust. For. 10:45-50.
Keyes R M, C C Grier (1981) Above-and below-ground net production in 40-year-old Douglas-fir stands on low and high productivity sites. Can. J. For. Res.11:599-605.

Kormanik P P, J L Ruehle, H D Muse (1990) Frequency distribution and heritability of first-order lateral roots in loblolly pine seedlings. For. Sci. 36:802-814.

Kozlowski T T, J P Kramer, G P Stephen (1991) The physiological ecology of woody plants. Academic Press. New York.USA. $657 \mathrm{p}$.

Ledig F T, M A Capó-Arteaga, P D Hodgskiss, H Shay, C FloresLopez, M T Conkle, B Bermejo-Velazquez (2001) Genetic diversity and mating system of a rare Mexican piñon, Pinus pinceana, and a comparison with Pinus maximartinezii (Pinaceae). Am. J. Bot. 88:1977-1987.

Li B, H L Allen, S E McKeand (1991) Nitrogen and family effects on biomass allocation of loblolly pine seedlings. For. Sci. 37:271-283.

Molina-Freaner, F P Delgado, D Piñero, N Perez-Nasser, E AlvarezBuylla (2001) Do rare pines need different conservation strategies? Evidence from three Mexican species. Can. J. Bot. 79:131-138.

Perry J P Jr (1991) The pines of Mexico and Central America. Timber Press, Portland, Oregon, USA. 231 p.

Pritchett W L (1991) Suelos Forestales. Propiedades, Conservación y Mejoramiento. Ed. Limusa. México. 634 p.

Rajkai V K (1991) Effect of soil water and nutriment supply on root characteristics and nutrient uptake of plants. In: Plant Roots and their Environment. B L McMichael, H Persson (eds). Proc. of ISRR-symposium August 21st-26th. 1988 Uppsala, Sweden. Elsevier. 649 p.

Salazar G, J J Vargas, J Jasso, G Molina, C Ramírez, J López (1999) Variación en el patrón de crecimiento en altura de cuatro especies de Pinus en edades tempranas. Madera y Bosques 5:19-34.

SAS Institute (1987) SAS / STAT Guide for Personal Computers. Version 6. SAS Institute Inc. Cary, N. C. 1028 p.

Wang J R, D B Hawkins, T Letchford (1998) Photosynthesis, water and nitrogen use efficiencies of four paper birch (Betula papyrifera) populations grown under different soil moisture and nutrient regimes. For. Ecol. Manage. 112:233-244.

Yoav W, A Eshel, U Kafkefi (1993) Plant roots. 2nd ed. Marcel Dekker Inc. New York. 1002 p.

Zhang J, J D Marshall, L Fins (1996) Correlated population differences in dry matter accumulation, allocation, and water-use efficiency in three sympatric conifer species. For. Sci. 42:242-249. 